\title{
A REMARK ON A THEOREM OF SERGE BERNSTEIN
}

EARL J. MICKLE

1. Introduction. In this note we shall discuss the following theorem of Bernstein (Math. Zeit. vol. 26 (1927) pp. 551-558).

THEOREM. Let $f(x, y)$ be a real-valued function which satisfies the following conditions.

(a) $f(x, y)$ is of class $C^{\prime \prime}$ in the entire $x y$-plane, that is, $f(x, y)$ is defined and has continuous partial derivative of the second order in the entire $x y$-plane.

(b) $f_{x x} f_{y y}-f_{x y}^{2} \leqq 0, f_{x x} f_{y y}-f_{x y}^{2} \not \equiv 0$.

Then $f(x, y)$ is not bounded.

We shall now review one of the main steps in the proof of this theorem. We can assume without loss of generality that $f(x, y)$ satisfies the further conditions:

(c) $f(0,0)=0, f_{x}(0,0)=0, q_{0}=f_{y}(0,0)>0, f_{x x}(0,0) f_{y y}(0,0)-f_{x y}^{2}(0,0)$ $<0$. The proof of the above theorem depends on the following lemma.

LEMMA 1.1. Let $f(x, y)$ be a real-valued function which satisfies the above conditions (a), (b), (c) and assume that there is a constant $h>0$ such that $-h<f(x, y)<h$. Then the set of points where the function $g(x, y)=f(x, y)-q_{0} y$ is different from zero contains a component $D$ which lies in the strip $-h / q_{0}<y<h / q_{0}$ and is bounded on either the right or the left.

E. Hopf noted that the proof given of this lemma does not rule out the case where each of the components which lies in the strip $-h / q_{0}$ $<y<h / q_{0}$ might oscillate back and forth so that points arbitrarily far from the origin in both directions occur in it. Hopf (Bull. Amer. Math. Soc. vol. 56 (1950) pp. 80-85) then gives a correct proof of the above theorem. In this note we give an alternate and somewhat simpler proof. We shall show that the argument of Bernstein can be used to prove the above theorem by the use of the following lemma, the proof of which is given in $\$ 3$.

LEMMA 1.2. Let $f(x, y)$ be a real-valued function which satisfies the above conditions (a), (b), (c) and assume that there is a constant $h>0$ such that $-h<f(x, y)<h$. Then there exists a real-valued function $f^{*}(x, y)$ which satisfies the following conditions.

Received by the editors October 1, 1949. 
$\left(a^{*}\right) f^{*}(x, y)$ is of class $C^{\prime \prime}$ in the entire $x y$-plane.

(b*) $f_{x x}^{*} f_{y y}^{*}-f_{x y}^{* 2} \leqq 0$.

$\left(\mathrm{c}^{*}\right) f^{*}(0,0)=f_{x}^{*}(0,0)=0, f_{y}^{*}(0,0)=f_{y}(0,0)=q_{0}, f_{x x}^{*}(0,0)=f_{x x}(0,0)$, $f_{x y}^{*}(0,0)=f_{x y}(0,0), f_{v y}^{*}(0,0)=f_{y y}(0,0)$.

$\left(\mathrm{d}^{*}\right)$ There is an $\eta>0$ such that $\left|f(x, y)-f^{*}(x, y)\right|<\eta$.

$\left(\mathrm{e}^{*}\right)$ The set of points where $g^{*}(x, y)=f^{*}(x, y)-q_{0} y$ is different from zero contains a component $D^{*}$ which lies in the strip $-h / q_{0}<y<h / q_{0}$ and is bounded on either the right or the left.

The argument of Bernstein can then be used to show that $f^{*}(x, y)$ can not be bounded, and hence by $\left(d^{*}\right)$ that $f(x, y)$ can not be bounded.

2. Preliminary lemma. Let $G(x, y ; \lambda)$ be a one-parameter family of real-valued functions which satisfy the following conditions for $-\eta<\lambda<\eta, \eta>0$.

C. $G(x, y ; \lambda)$ is of class $C^{\prime \prime}$ in the entire $x y$-plane.

C. $G_{x x} G_{y y}-G_{x y}^{2} \leqq 0$.

$\mathrm{C}_{3} . G(0,0 ; \lambda)=G_{x}(0,0 ; \lambda)=G_{y}(0,0 ; \lambda)=0$.

C. $G_{x x}(0,0 ; \lambda), G_{x y}(0,0 ; \lambda), G_{y y}(0,0 ; \lambda)$ are constant and $G_{x x}(0,0$; ג) $G_{y y}(0,0 ; \lambda)-G_{x y}^{2}(0,0 ; \lambda)<0$.

$\mathrm{C}_{5}$. There is a number $\alpha>0$ such that $G(x, \alpha ; 0)<0, G(x,-\alpha ; 0)>0$ for $-\infty<x<\infty$.

C6. $G\left(x, y ; \lambda_{1}\right)<G\left(x, y ; \lambda_{2}\right)$ for $\lambda_{1}<\lambda_{2},(x, y) \neq(0,0)$.

Following the argument of Bernstein we have the following facts. Under the above conditions there exist two numbers $k_{1}, k_{2}$ depending upon $G_{x x}(0,0 ; \lambda), G_{x y}(0,0 ; \lambda), G_{y y}(0,0 ; \lambda)$ (see $\left.\mathrm{C}_{4}\right)$ and a number $\epsilon(\lambda)>0$ such that $G(x, y ; \lambda)>0$ for $y=k_{1} x, 0<|x|<\epsilon(\lambda)$ and $G(x, y ; \lambda)$ $<0$ for $y=k_{2} x, 0<|x|<\epsilon(\lambda)$. Let $\gamma_{1}(\lambda)$ be the segment $y=k_{1} x$, $0<x<\epsilon(\lambda), \gamma_{3}(\lambda)$ be the segment $y=k_{1} x,-\epsilon(\lambda)<x<0, \gamma_{2}(\lambda)$ be the segment $y=k_{2} x, 0<x<\epsilon(\lambda)$, and $\gamma_{4}(\lambda)$ be the segment $y=k_{2} x,-\epsilon(\lambda)$ $<x<0$. For each $\lambda$ the set of points in the $x y$-plane where $G(x, y ; \lambda)$ $\neq 0$ contains four distinct components $D_{i}(\lambda) \supset \gamma_{i}(\lambda), i=1,2,3,4$. For the components $D_{i}(\lambda)$

$$
\begin{aligned}
& G(x, y ; \lambda)>0 \text { for }(x, y) \in D_{1}(\lambda) \text { or } D_{3}(\lambda), \\
& G(x, y ; \lambda)<0 \text { for }(x, y) \in D_{2}(\lambda) \text { or } D_{4}(\lambda),
\end{aligned}
$$

and, by condition $C_{6}$, for the closures $c\left[D_{i}(\lambda)\right]$ of $D_{i}(\lambda)$ we have the relations

$$
\begin{array}{ll}
c\left[D_{i}\left(\lambda_{1}\right)\right]-(0,0) \subset D_{i}\left(\lambda_{2}\right), & \lambda_{1}<\lambda_{2}, i=1,3, \\
c\left[D_{i}\left(\lambda_{2}\right)\right]-(0,0) \subset D_{i}\left(\lambda_{1}\right), & \lambda_{1}<\lambda_{2}, i=2,4 .
\end{array}
$$


From (2) it thus follows that

$$
{ }_{c}\left[D_{1}(\lambda)\right]_{c}\left[D_{3}(\lambda)\right]=C\left[D_{2}(\lambda)\right] c\left[D_{4}(\lambda)\right]=(0,0) .
$$

We now prove the following preliminary lemma.

LEMMA 2.1. Let $G(x, y ; \lambda)$ be a one-parameter family of real-valued functions which satisfy the above conditions $\mathrm{C}_{1}, \cdots, \mathrm{C}_{6}$. Then there is a number $\lambda_{0},-\eta<\lambda_{0}<\eta$, such that the set of points in the $x y$-plane where $G\left(x, y ; \lambda_{0}\right) \neq 0$ contains a component which lies in the strip $-\alpha<y<\alpha$ and is bounded on either the right or the left.

Proof. Consider the components $D_{i}(\lambda)$ described above.

Case 1 . One of the components $D_{i}(0)$, say $D_{i_{0}}(0)$, does not cross the line $x=x_{0}$. By condition $C_{b}, D_{i_{0}}(0)$ does not cross either of the lines $y= \pm \alpha$ (otherwise it would have to contain the entire line and hence would cross the line $\left.x=x_{0}\right)$. Thus $D_{i_{0}}(0)$ lies in the strip $-\alpha<y<\alpha$ and is bounded on either the right or the left. Take $\lambda_{0}=0$ in this case.

Case 2. Every component $D_{i}(0), i=1,2,3,4$, crosses every line $x=x_{0},-\infty<x_{0}<\infty$. Set

$$
u_{i}=\operatorname{lub} y, l_{i}=\text { glb } y \text { for }(1, y) \in D_{i}(0),-\alpha<y<\alpha, i=1,2,3,4 \text {, }
$$

and let

$$
u=\max u_{1}, u_{2}, u_{3}, u_{4}, \quad l=\min l_{1}, l_{2}, l_{3}, l_{4} \text {. }
$$

Case $2_{1} . u=u_{i_{1}}, u \neq u_{i}$ for $i \neq i_{1}$. Then $l=l_{i_{2}}$ and by relations (3) there are at least two integers $i$ such that $l_{i} \neq l$ and for at least one of them, say $i_{3}, i_{3} \neq i_{1}$. Then

$$
-\alpha \leqq l_{i_{3}}<l_{i_{3}}<u_{i_{3}}<u_{i_{1}} \leqq \alpha .
$$

By (4) we have a point $\left(1, y_{1}\right) \in D_{i_{1}}(0), u_{i_{3}}<y_{1}<u_{i_{1}}$ and a point $\left(1, y_{2}\right) \in D_{i_{3}}(0), l_{i_{2}}<y_{2}<l_{i_{3}}$. Let $\gamma^{i}$ be a simple arc with end points $(0,0)$ and $\left(1, y_{j}\right)$ and lying in $D_{i_{j}}(0)+(0,0), j=1,2$. Then there exist numbers $x_{1}<x_{2}$ such that $\gamma^{1}$ and $\gamma^{2}$ lie between the lines $x=x_{1}$ and $x=x_{2}$. If $D_{i_{3}}(0)$ contains a point on the line $x=x_{1}$ and a point on the line $x=x_{2}$, then a simple arc joining them and lying in $D_{i_{3}}(0)$ would have to cross either $\gamma^{1}$ or $\gamma^{2}$. This is impossible. Hence $D_{i_{3}}(0)$ satisfies the conditions of Case 1 and Case $2_{1}$ is impossible.

Case $2_{2} . l=l_{i_{1}}, l_{i} \neq l$ for $i \neq i_{1}$. The same reasoning as that used in Case $2_{1}$ shows that Case $2_{2}$ is impossible.

Case $2_{3} . u=u_{i_{1}}=u_{i_{3}}, i_{1} \neq i_{2}$ and $l=l_{i_{3}}=l_{i_{4}}, i_{3} \neq i_{4}$. Then $-\alpha<l<u$ $<\alpha$ and each component $D_{i}(0), i=1,2,3,4$, lies in the strip $-\alpha<y$ $<\alpha$. By the relations (3), $i_{1}, i_{2}$ are not both odd or both even integers and $i_{3}, i_{4}$ are not both odd or both even integers. By renumbering if 
necessary let $i_{1}$ and $i_{3}$ be the odd integers. Then $i_{2}$ is an even integer. Take $\lambda_{0}, 0<\lambda_{0}<\eta$. By $(2), D_{i_{2}}\left(\lambda_{0}\right) \subset D_{i_{2}}(0)$ and hence $D_{i_{2}}\left(\lambda_{0}\right)$ lies in the strip $-\alpha<y<\alpha$. By (2), $\left(1, u_{i_{1}}\right) \in D_{i_{1}}\left(\lambda_{0}\right),\left(1, l_{i_{3}}\right) \in D_{i_{3}}\left(\lambda_{0}\right)$, and

$$
l_{i_{3}}<y<u_{i_{1}}
$$

for $(1, y) \in D_{i_{2}}\left(\lambda_{0}\right)$.

Let $\gamma^{1}$ be a simple arc with end points $(0,0)$ and $\left(1, u_{i_{1}}\right)$ and lying in $D_{i_{1}}\left(\lambda_{0}\right)+(0,0)$, and let $\gamma^{2}$ be a simple arc with end points $(0,0)$ and $\left(1, l_{i_{8}}\right)$ and lying in $D_{i_{3}}\left(\lambda_{0}\right)+(0,0)$. By (5) the same reasoning as that used in Case $2_{1}$ shows that $D_{i_{2}}\left(\lambda_{0}\right)$ is bounded on either the right or the left.

3. Proof of Lemma 1.2. Let $f(x, y)$ be a real-valued function which satisfies the conditions (a), (b), (c) of $\$ 1$ and assume that there is a constant $h>0$ such that $-h<f(x, y)<h$. By condition (c) there is a number $a \neq 0$ such that

$$
f_{x x} f_{y y}-f_{x y}^{2}<0 \quad \text { for } x^{2}+y^{2} \leqq a^{2} .
$$

For this number $a \neq 0$ set

$$
\phi(x, y)= \begin{cases}(t-\sin t) / 2 \pi, \quad t=2 \pi\left(x^{2}+y^{2}\right) / a^{2}, & \text { for } x^{2}+y^{2} \leqq a^{2}, \\ 1 & \text { for } x^{2}+y^{2}>a^{2} .\end{cases}
$$

Then $\phi(x, y)$ satisfies the following conditions. (i) $\phi$ is of class $C^{\prime \prime}$ in the entire $x y$-plane. (ii) $\phi(0,0)=0$. (iii) $\phi_{x}=\phi_{y}=\phi_{x x}=\phi_{x y}=\phi_{y y}=0$ for $(x, y)=(0,0)$ and for $x^{2}+y^{2} \geqq a^{2}$. (iv) $0<\phi(x, y) \leqq 1$ for $(x, y) \neq(0,0)$. Elementary computations show that in view of (6) and the conditions (i), (ii), (iii), (iv) satisfied by $\phi$ there is a number $\eta>0$ such that the functions

$$
F(x, y ; \lambda)=f(x, y)+\lambda \phi(x, y), \quad-\eta<\lambda<\eta,
$$

satisfy the following conditions.

(a') $F(x, y ; \lambda)$ is of class $C^{\prime \prime}$ in the entire $x y$-plane.

(b') $F_{x x} F_{y y}-F_{x y}^{2} \leqq 0$.

(c') $F(0,0 ; \lambda)=0, F_{x}(0,0 ; \lambda)=0, F_{y}(0,0 ; \lambda)=f_{y}(0,0)=q_{0}, F_{x x}(0,0 ;$ $\lambda)=f_{x x}(0,0), F_{x y}(0,0 ; \lambda)=f_{x y}(0,0), F_{y y}(0,0 ; \lambda)=f_{y y}(0,0)$.

$\left(\mathrm{d}^{\prime}\right)|f(x, y)-F(x, y ; \lambda)|<\eta$.

Then, for $\alpha=h / q_{0}$, the functions

$$
G(x, y ; \lambda)=F(x, y ; \lambda)-q_{0} y, \quad-\eta<\lambda<\eta,
$$

satisfy the assumptions of Lemma 2.1. Hence for the number $\lambda_{0}$, $0 \leqq \lambda_{0}<\eta$, determined in Lemma 2.1 , the function $f^{*}(x, y)=F\left(x, y ; \lambda_{0}\right)$ satisfies the conditions of Lemma 1.2.

The Ohio State University 